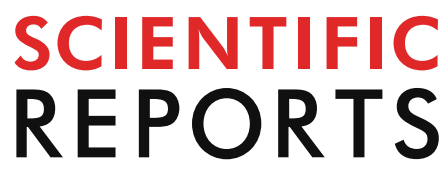

natureresearch

Check for updates

\title{
Study of the hydrogen
} physisorption on adsorbents based on activated carbon by means of statistical physics formalism: modeling analysis and thermodynamics investigation

\begin{abstract}
Manel Ben Yahia ${ }^{1 \bowtie}$ \& Sarra Wjihi ${ }^{2 \bowtie}$
An advanced statistical physics model has been applied to study the hydrogen adsorption isotherm on two modified types of activated carbon, namely granular coal activated carbon (AC (GC)) and coconut shell activated carbon (AC (CS)). This model is established with the statistical physics approach. It is a more general model including various parameters having a defined physico-chemical sense which were discussed at different temperatures. Hence new physic-chemical interpretations of the adsorption process of hydrogen are provided. The analysis of the hydrogen uptake capacities at saturation showed that the AC (GC) adsorbent displayed a high adsorption capacity $(3.21 \mathrm{mg} / \mathrm{g})$. This due to the contribution of the number of hydrogen molecules per site (1.27) associated with the receptor sites density $(0.74 \mathrm{mg} / \mathrm{g}$ ) and the number of formed layers (3.42). The modeling results suggested that the hydrogen adsorption occurred by non-parallel positions on the two tested adsorbents thus evincing that the adsorption cannot be other than a multi-molecular process. The calculated adsorption energies globally varied from 7.01 to $12.92 \mathrm{~kJ} / \mathrm{mol}$, confirming the physical nature of the adsorption process for both studied systems. The thermodynamic functions, namely internal energy, enthalpy and entropy were estimated to better analyze the hydrogen sorption process. In summary, the statistical physics analysis provided reliable concrete physico-chemical interpretations of hydrogen adsorption process on carbon-based adsorbents with various microstructures to develop a storage compounds with a suitable framework for a hydrogen storage structure.
\end{abstract}

The growth of the world population and the technology advancement solicit an increasing energy demand which is based on a massive use of fossil fuels ${ }^{1}$. But their use poses two major problems. The first is a problem of availability with the continuous decrease of the global reserves of fossil fuels. The second is that the resulting greenhouse gas emissions are high and strongly contribute to global warming. It is found that hydrogen, the most abundant element in the universe, is the uncontested candidate to play a key role in the development of a new energy system, both safe and compact. However, the optimal performance of such an energy source requires the development of new storage methods. The physical storage method for hydrogen adsorption on porous structures is the best method to use for storing hydrogen in comparison with other techniques. Moreover, porous materials offer numerous advantages for the storage of hydrogen thanks to the fast hydrogen uptake and release kinetics ${ }^{2-5}$. One of the porous materials is activated carbon which offers good chemical stability, easy reversibility, easy availability and low price ${ }^{6}$. Porous carbons can be synthesized from different carbon precursors, including wood, peat, coal, coconut and fruit stones and polymers, by physical and chemical activation methods. On this basis, coals have become the principal precursor for the preparation of activated carbons due

${ }^{1}$ Physics Department, Rabigh College of Science and Arts, King Abdulaziz University, Jeddah, P.O Box 344, Rabigh 21911, Saudi Arabia. 'Laboratory of Quantum and Statistical Physics, LR18ES18, Faculty of Science of Monastir, Monastir University, Monastir, Tunisia. ${ }^{\boxplus}$ email: mbinyhea@kau.edu.sa; sarawjihi@yahoo.fr 
to their intrinsic microstructure and surface chemical characteristics ${ }^{7,8}$. To better explore and understand the physisorption mechanism of hydrogen on activated carbon adsorbents systems, the adsorption isotherms have been studied and interpreted. In this respect, various theoretical studies using adsorption models ranging from Langmuir, Langmuir-Freundlich and Tóth models have been realized ${ }^{9,10}$. A comparison between these models for a similar system led us to conclude that almost all of them are empirical. Hence, all their parameters are mathematical and they have no physical meaning. Their values are in relation with mathematical parameters such as a slope of the curve, its origin value, etc. None of the models used in the literature used our statistical approach, especially the particular parameters introduced into our models, such as the number of hydrogen molecules per site, the ancrage number, and the individual sorption energy. We notice that the only information deduced from some articles is the molar adsorption energy and the number of sites, sometimes synthesized empirically. The objective of this project is to provide a statistical physics framework to simulate the hydrogen adsorption isotherms of two activated carbon adsorbents [granular coal activated carbon (AC (GC)) and coconut shell activated carbon $(\mathrm{AC}(\mathrm{CS}))]^{11}$. The goal of this statistical physics approach is to allow the development of many models which are able to adjust the hydrogen sorption isotherms. These models based on statistical physics have allowed an estimation of several physicochemical parameters in good relation with the sorption mechanism. The main advantage of our modeling analysis is to provide new microscopic and macroscopic information included in the sorption phenomenon that did not arise in any case of all previous works and models. In our research, we can present steric parameters like the total saturation adsorption quantities, the numbers of captured hydrogen molecules per binding site and the receptor site densities. These steric parameters could not be determined by means of classical models.

\section{Experimental methods}

Materials. The characterization and preparation of the two types of activated carbon (AC (GC) and AC (CS)) are discussed $\mathrm{in}^{11}$. The physical activation of $\mathrm{AC}(\mathrm{GC})$ and $\mathrm{AC}(\mathrm{CS})$ was performed in a vertical activation reactor placed inside an oven. The carbonization and oxidation are part of the production processes. As a first step, the granular coal and the coconut shell were heat treated by flowing $\mathrm{O}_{2}$ at $573 \mathrm{~K}$ and $100 \mathrm{~mL} / \mathrm{min}$ during $6 \mathrm{~h}$.

Adsorption isotherms experiments. Hydrogen of $99.99 \%$ purity was used as adsorbate in the experiment. The experimental apparatus mainly consists of a charging cell (control volume) and a stainless steel measuring (or adsorption) cell with internal volumes of $1170 \mathrm{~mL}$ and $85.5 \mathrm{~mL}$, respectively. The charging and the adsorption cells are connected using a small tube. The apparatus is immersed in a temperature controlled water bath with a precision of $0.2 \mathrm{~K}$. The pressure of the two cells is measured using an absolute pressure transmitter (Druck, PTX 1400), with a pressure range of 0 to $40 \mathrm{MPa}$ and a full scale uncertainty of $0.15 \%$. Temperature is measured using thermocouples of class-A and type K. A thermocouple is installed in the measuring cell and is in contact with the adsorbent in the adsorption cell. This allows to obtain with precision a direct temperature of the adsorbent. The pressures and temperatures are recorded using a data logger with a sampling interval of $10 \mathrm{~s}$. The detailed experimental procedures and the experimental setup used to prepare hydrogen adsorption measurement were previously described by Nasruddin et al. ${ }^{11}$.

\section{Description of the hydrogen adsorption behavior and development of the multilayer model with saturation}

A general analysis of the experimental hydrogen adsorption data indicated that the temperature had a minor impact on the evolution of hydrogen uptake capacities (see Fig. 1) where practically the same uptake capacity was found at all the operating temperatures. It should be noted that the adsorption isotherms indicated the occurrence of an exothermic process where a slight decrease of the hydrogen adsorption capacity was shown with respect to temperature. In addition, the adsorption patterns did not reach a saturation condition at the highest investigated $\mathrm{H}_{2}$ partial pressure, thus hypothesizing that the adsorption could be associated to a variable number of adsorbed layers. A generalized statistical physics model was established to explore the hydrogen adsorption phenomenon and to provide a theoretical interpretation. This statistical physics model assumed that the sorption of the hydrogen molecules on the two employed adsorbents occurred via the creation of a variable number of layers, driven by two adsorption energies, which are mainly associated with these interactions: interactions between $\mathrm{H}_{2}$ and activated carbon based adsorbent, and interactions between $\mathrm{H}_{2}$ molecules (between $\mathrm{N}$ formed layers). It is worth noting that this model was developed by considering simple hypotheses. In a first approach, the gaseous $\mathrm{H}_{2}$ molecules were considered as an ideal gas, since the interaction of the hydrogen molecules is reduced to an average constant interaction represented by the chemical potential. Furthermore, the internal degrees of freedom of hydrogen are lost during sorption and only the degree of translation has been taken. This is explained by the fact that the electronic degree of freedom cannot be thermally excited. In addition, the vibrational degree of freedom can be discarded in comparison to the translational degree ${ }^{12,13}$. This advanced model was developed through the grand canonical ensemble in statistical physics. The expression of the grand canonical partition function of a single site is given by:

$$
z_{g c}=1+e^{\beta\left(\varepsilon_{1}+\mu\right)}+e^{\beta\left(\varepsilon_{1}+\varepsilon_{2}+2 \mu\right)} \frac{1-e^{\beta\left(\varepsilon_{2}+\mu\right)^{N}}}{1-e^{\beta\left(\varepsilon_{2}+\mu\right)}}
$$

where $\left(-\varepsilon_{1}\right)$ and $\left(-\varepsilon_{2}\right)$ are the receptor site adsorption energy, $\mu$ is the chemical potential and $\beta$ is defined as $1 /$ $\mathrm{k}_{\mathrm{B}} \mathrm{T}$, where $\mathrm{k}_{\mathrm{B}}$ is the Boltzmann constant and $\mathrm{T}$ is the absolute temperature. 

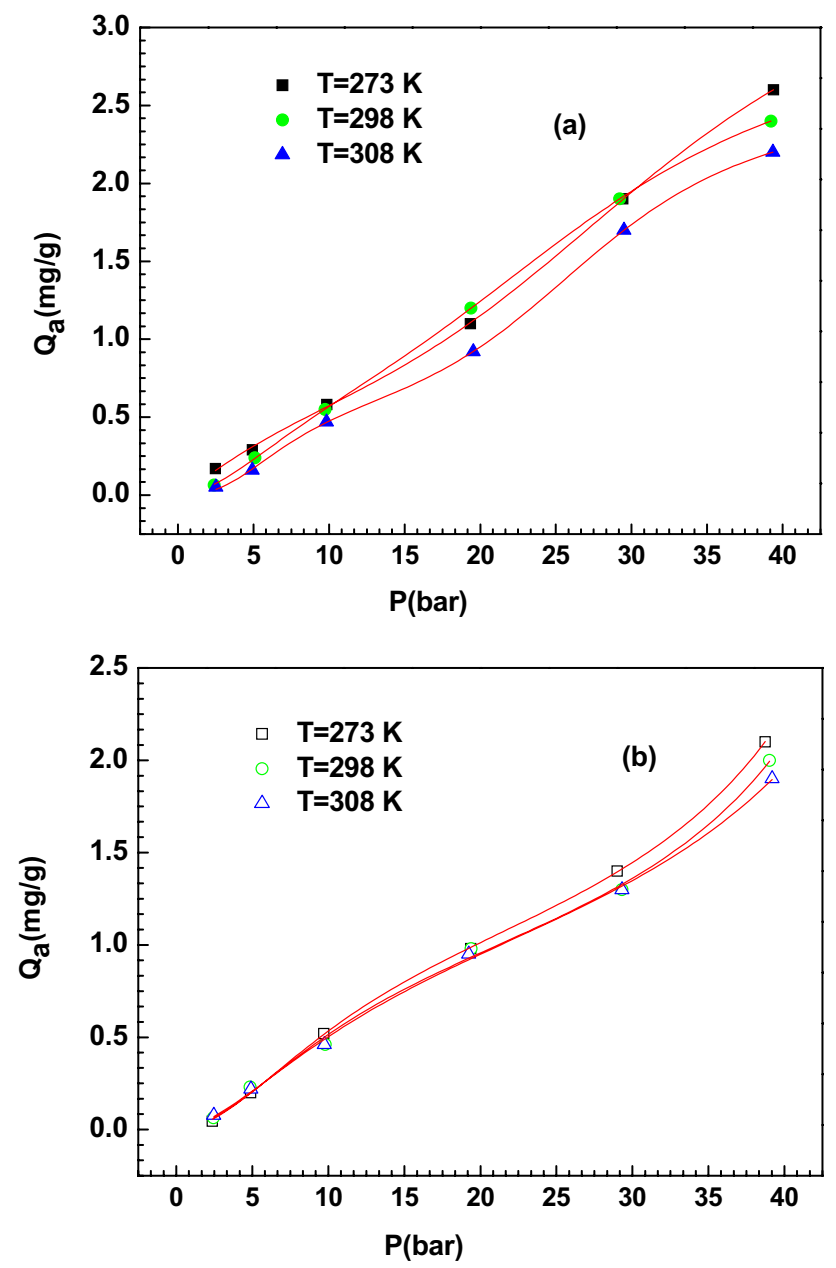

Figure 1. Adsorption isotherms of hydrogen on (a) Granular Coal Activated Carbon (AC (GC)) and (b) Coconut Shell Activated Carbon (AC (CS)), at different temperature and fitting by multilayer statistical physics model (MLS).

A non-fixed number of hydrogen molecules were considered to adsorb on $\mathrm{D}_{\text {sr }}$ binding sites per mass unit of the AC adsorbents. The total grand canonical partition function associated with these binding sites, is supposed to be identical and independent and is given as follows:

$$
Z_{\mathrm{gc}}=\left[1+\mathrm{e}^{\beta\left(\varepsilon_{1}+\mu\right)}+\mathrm{e}^{\beta\left(\varepsilon_{1}+\varepsilon_{2}+2 \mu\right)} \frac{1-\mathrm{e}^{\beta\left(\varepsilon_{2}+\mu\right)^{N}}}{1-\mathrm{e}^{\beta\left(\varepsilon_{2}+\mu\right)}}\right]^{D_{s r}}
$$

The average site occupation number can be written as ${ }^{14}$ :

$$
\mathrm{N}_{\mathrm{O}}=\mathrm{k}_{\mathrm{B}} \mathrm{T} \frac{\partial \ln \mathrm{Z}_{\mathrm{gc}}}{\partial \mu}=D_{s r} \mathrm{k}_{\mathrm{B}} \mathrm{T} \frac{\partial \ln \mathrm{z}_{\mathrm{gc}}}{\partial \mu}
$$

Generally, the adsorption of $\mathrm{H}_{2}$ gaseous molecules onto a binding site (S) should incorporate a stoichiometric coefficient $\mathrm{n}_{\mathrm{ms}}$ as depicted in the following equation:

$$
n_{m s} \mathrm{H}_{2}+S \leftrightarrow S \mathrm{H}_{2 n_{m s}}
$$

When the thermodynamic equilibrium is reached according to Eq. (4), the equality of the different chemical potentials can be given by: $\mu_{\mathrm{m}}=\mu / \mathrm{n}_{\mathrm{ms}}$, where $\mu$ is the chemical potential of the sorbed molecules, $\mathrm{n}_{\mathrm{ms}}$ the number of hydrogen molecules per binding site and $\mu_{\mathrm{m}}$ is the chemical potential of the hydrogen gaseous molecules. By utilizing the ideal gas approximation, the chemical potential in the gaseous state can be given by the following equation $^{15}$ :

$$
\mu_{\mathrm{m}}=\mathrm{k}_{\mathrm{B}} \mathrm{T} \ln \frac{\mathrm{N}}{\mathrm{Z}_{\mathrm{g}}}
$$




\begin{tabular}{|l|l|l|}
\hline Name of tested model & Partition function & Reference \\
\hline Monolayer with one energy (MLO) & $Z_{\mathrm{gc}}=\left(1+\mathrm{e}^{\beta\left(\varepsilon_{1}+\mu\right)}\right)^{D_{s r}}$ & 15,16 \\
\hline Double layer with two energies (DLT) & $Z_{\mathrm{gc}}=\left(1+\mathrm{e}^{\beta\left(\varepsilon_{1}+\mu\right)}+\mathrm{e}^{\beta\left(\varepsilon_{1}+\varepsilon_{2}+2 \mu\right)}\right)^{D_{s r}}$ & 15,16 \\
\hline Multilayer with saturation (MLS) & $Z_{\mathrm{gc}}=\left(1+\mathrm{e}^{\beta\left(\varepsilon_{1}+\mu\right)}+\mathrm{e}^{\beta\left(\varepsilon_{1}+\varepsilon_{2}+2 \mu\right)} \frac{1-\mathrm{e}^{\beta\left(\varepsilon_{2}+\mu\right)^{N}}}{1-\mathrm{e}^{\beta\left(\varepsilon_{2}+\mu\right)}}\right)^{D_{s r}}$ & \\
\hline
\end{tabular}

Table 1. Partition functions of the proposed models.

where $\mathrm{Z}_{\mathrm{g}}=\mathrm{Z}_{\mathrm{gtr}}$ is the translation partition function of the hydrogen gaseous molecules which can be written as $^{15,16}$ :

$$
\mathrm{Z}_{\mathrm{g}}=\mathrm{Z}_{\mathrm{gtr}}=\mathrm{V}\left(\frac{2 \pi \mathrm{mk}_{\mathrm{B}} \mathrm{T}}{\mathrm{h}^{2}}\right)^{3 / 2}
$$

where $h, V$, and $m$ are the Planck constant, the volume of the hydrogen gas and the sorbed molecule mass, respectively. The translation partition function per unit of volume $\mathrm{z}_{\mathrm{gtr}}$, for an ideal gas, can be represented as a function of the hydrogen saturated vapor pressure $\mathrm{P}_{\mathrm{vs}}$ and the hydrogen vaporization energy $\Delta \mathrm{E}^{\mathrm{v}}$ by the equation ${ }^{14-19}$ :

$$
Z_{\text {gtr }}=\beta \mathrm{P}_{\mathrm{vs}} \mathrm{e}^{\frac{\Delta \mathrm{E}^{\mathrm{v}}}{\mathrm{RT}}}
$$

where

$$
\mathrm{P}_{\mathrm{vs}}=\left(\frac{\left(2 \pi \mathrm{mk}_{\mathrm{B}} \mathrm{T}\right)^{3 / 2}}{\mathrm{~h}^{3}}\right)\left(k_{\mathrm{B}} \mathrm{T}\right) \mathrm{e}^{-\frac{\Delta \mathrm{E}^{\mathrm{v}}}{\mathrm{RT}}}
$$

Using Eq. (4) and the number of average site occupation $\mathrm{N}_{\mathrm{o}}$, the average number of sorbed molecules is written as:

$$
\mathrm{Q}_{\mathrm{a}}=\mathrm{n}_{m s} \mathrm{~N}_{\mathrm{o}}
$$

The adsorbed quantity is written as:

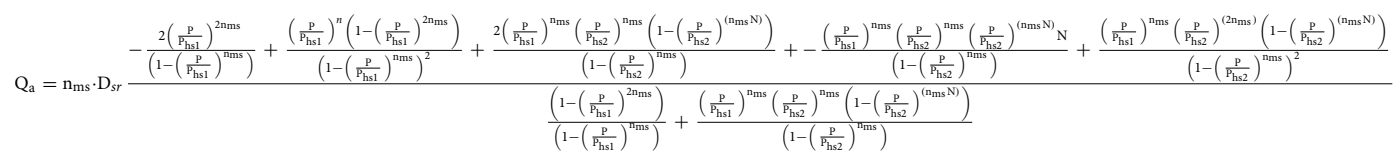

The theoretical equation of this multilayer model presents numerous physicochemical parameters that can be determined from the experimental data of hydrogen adsorption on the two ACs by modeling examination: the number of hydrogen molecules per binding site $\mathrm{n}_{\mathrm{ms}}$, the density of binding sites $\mathrm{D}_{\mathrm{sr}}$, the number of sorbed layers $\mathrm{N}$ and the pressures at half saturation $\mathrm{P}_{\mathrm{hs} 1}$ and $\mathrm{P}_{\mathrm{hs} 2}$ for the first and the Nth layers respectively.

\section{Results and discussion}

Simulation: Levenberg-Marquardt iterating algorithm. A computer simulation was used to adjust the hydrogen adsorption isotherm with the proposed models. All the hydrogen sorption isotherms were adjusted by three statistical physics models; multilayer model with saturation (MLS), double layer model with two energies (DLT) and single layer model with one energy (MLO) using microcal origin software (Origin Lab, Northampton, MA). In Table 1, the partition functions of the three proposed models are shown. In this paper, two adjusting parameters were employed: the determination coefficient $\mathrm{R}^{2}$ and the residual root mean square error (RMSE). It should be noted that a sorption isotherm is in a good correlation with the tested statistical physics model, when the coefficient of adjustment $\mathrm{R}^{2}$ tends to the unit and the values of residual root mean square RMSE are close to zero. All the values of the determination coefficient $\left(\mathrm{R}^{2}\right)$ and RSME of all the established models, as adjusted to the adsorption isotherm of hydrogen on AC (GC) and AC (CS), are summarized in Table 2. As can be observed, the multilayer adsorption model presents the perfect adjusting results since the values of RMSE are close to zero (from 0.009 to 0.012 ) and the values of $\mathrm{R}^{2}$ are close to unity (0.9982-0.9995) in comparison with the other applied models. Consequently, it was adopted for the analysis and discussion of the hydrogen adsorption process. The fitting of the adsorption isotherms of hydrogen on the two ACs using the multilayer model with saturation is illustrated in Fig. 1. We also present in Table 3 the adjusted values of the parameters of the selected model i.e. the hydrogen molecules per binding site $\mathrm{n}_{\mathrm{ms}}$, the receptor sites density $\mathrm{D}_{\mathrm{sr}}$, the hydrogen uptake capacity at saturation $\mathrm{Q}_{\text {asat }}$ and the estimated values of adsorption energies $-\varepsilon_{1}$ and $-\varepsilon_{2}$.

Interpretation of the parameters of statistical physics models. The adsorption of hydrogen on AC (GC) and AC (CS) at three operating temperatures was interpreted and analyzed via the adjusted values of the different parameters in terms of steric and energetic points of view.

Steric analysis. The hydrogen adsorption capacity at saturation $\left(\mathrm{Q}_{\text {asat }}\right)$. The adopted statistical physics model was able to determine the values of the uptake capacity at saturation at different temperatures and to provide a 


\begin{tabular}{|l|l|l|l|l|}
\hline \multirow{4}{*}{ Adsorbent } & \multirow{4}{*}{ T (K) } & MLo & DLT & MLS \\
\hline $\mathbf{R}^{2}$ & 273 & 0.9721 & 0.9893 & 0.9993 \\
\hline \multirow{4}{*}{ AC (GC) } & 298 & 0.9781 & 0.9831 & 0.9995 \\
\cline { 2 - 5 } & 308 & 0.9657 & 0.9842 & 0.9982 \\
\hline \multirow{5}{*}{ RMSE } & 273 & 0.371 & 0.105 & 0.012 \\
\hline \multirow{3}{*}{ AC (CS) } & 298 & 0.353 & 0.145 & 0.035 \\
\cline { 2 - 5 } & 308 & 0.497 & 0.124 & 0.009 \\
\hline
\end{tabular}

Table 2. Values of coefficient of determination $\mathrm{R}^{2}$ and RMSE of each model.

\begin{tabular}{|c|c|c|c|c|c|c|c|}
\hline \multirow[b]{2}{*}{ Adsorbent } & \multirow[b]{2}{*}{ Temperature (K) } & \multicolumn{6}{|c|}{ Parameters } \\
\hline & & $\mathbf{n}_{\mathrm{ms}}$ & $\mathrm{D}_{\mathrm{sr}}(\mathrm{mg} / \mathrm{g})$ & $\mathbf{N}_{\mathrm{T}}$ & $Q_{\text {asat }}(\mathrm{mg} / \mathrm{g})$ & $-\varepsilon_{1}(\mathbf{k J} / \mathrm{mol})$ & $-\varepsilon_{2}(\mathrm{~kJ} / \mathrm{mol})$ \\
\hline \multirow{3}{*}{$\mathrm{AC}(\mathrm{GC})$} & 273 & 1.27 & 0.74 & 3.42 & 3.21 & -10.25 & -7.44 \\
\hline & 298 & 1.86 & 0.43 & 3.76 & 3.00 & -11.88 & -8.89 \\
\hline & 308 & 2.62 & 0.23 & 4.13 & 2.48 & -12.92 & -9.47 \\
\hline \multirow{3}{*}{$\mathrm{AC}(\mathrm{CS})$} & 273 & 1.15 & 0.64 & 2.87 & 2.11 & -10.02 & -7.01 \\
\hline & 298 & 1.66 & 0.38 & 3.24 & 2.04 & -11.52 & -8.45 \\
\hline & 308 & 2.43 & 0.21 & 3.75 & 1.91 & -12.49 & -9.21 \\
\hline
\end{tabular}

Table 3. Estimated values of parameters of MLS model for the adsorption of hydrogen on AC (GC) and AC (CS).

clarification of the described profile of the hydrogen adsorption isotherms. We note that the expression of this parameter is obtained by the correlation of the three parameters of the saturated multilayer model: $Q_{\text {asat }}=n_{m s}$. $D_{\text {sr }}$ $(1+N)$. Table 3 shows that the trend of this parameter satisfies the following ranking: $Q_{\text {asat }}\left(H_{2}-A C(G C)\right)>Q_{\text {asat }}$ $\left(\mathrm{H}_{2}\right.$-AC (CS)). This result indicates that the AC (GC) exhibits the highest adsorption capacity of hydrogen molecules compared to the AC (CS) adsorbent.

This trend is mainly due to the specific beneficial characteristics of the AC (GC) adsorbent, such as well-developed internal pore structure, high surface area and high affinity of the binding sites of the AC (GC) surface ${ }^{6-8}$. In fact, according to the characterization data ${ }^{11}$, the $\mathrm{AC}(\mathrm{GC})$ has the largest surface area $\left(729.944 \mathrm{~m}^{2} / \mathrm{g}\right)$ in comparison with AC (CS) adsorbent $\left(414.900 \mathrm{~m}^{2} / \mathrm{g}\right)$. It is clear that this texture parameter is the main feature affecting the hydrogen adsorption amounts. Figure 2 reports the hydrogen adsorption capacities at saturation with respect to temperature for the two adsorbents. It can be observed that AC (GC) and AC (CS) presented a similar behavior in the slope of the curve, with a gradual decrease in the uptake capacity as a function of the different tested operating temperatures. This can be explained by the fact that the increase of temperature could improve the thermal collisions between the hydrogen molecules; therefore, the hydrogen adsorption binding on both ACs was decreased, which was related to an exothermic sorption mechanism.

The number of hydrogen molecules per site $\left(\mathrm{n}_{\mathrm{ms}}\right)$. The number of captured molecules per site plays a significant role to elucidate the hydrogen adsorption mechanism, thus the coefficient $\mathrm{n}_{\mathrm{ms}}$ is able to outline the adsorption orientation at different temperatures. In addition, it can determine the aggregation degree of hydrogen molecules. To describe the adsorption position, it is sufficient to compare the $\mathrm{n}_{\mathrm{ms}}$ adjusted values by the unity. Two cases can be examined: $n_{m s}$ values lower than unity, i.e. each binding site of the AC adsorbents can receive a part of the hydrogen molecules. Therefore, the adsorption position is parallel. In the second case, the $\mathrm{n}_{\mathrm{ms}}$ values are higher or equal to unity; each binding site can receive one or more hydrogen molecules. Table 3 shows that all the calculated values of this parameter were higher than unity at different operating temperature. Overall, the values of $\mathrm{n}_{\mathrm{ms}}$ varied from 1.27 to 2.89 and from 1.45 to 2.43 for the adsorption systems $\mathrm{H}_{2}-\mathrm{AC}(\mathrm{GC})$ and $\mathrm{H}_{2}-\mathrm{AC}(\mathrm{CS})$, respectively. This result demonstrated a non-parallel position of hydrogen on both AC adsorbents involving a multi-molecular process, thereby the hydrogen molecules could link to a single adsorption site. As stated, the aggregation degree of the hydrogen molecules can also be determined and interpreted by the parameter $\mathrm{n}_{\mathrm{ms}}$. The results showed that this aggregation process could happen practically at three tested temperatures where $\mathrm{n}_{\mathrm{ms}} \approx 1$ for the monomer, $\mathrm{n}_{\mathrm{ms}} \approx 2$ for the dimmer and $\mathrm{n}_{\mathrm{ms}} \approx 3$ for the trimmer for both AC adsorbents. We can deduce that a low aggregation degree indicates that the hydrogen adsorption system is not highly thermally activated. In other words, the temperature has a minor impact on the aggregation process. A comparison between the two adsorption systems indicated that this parameter varied as follows: $\mathrm{n}_{\mathrm{ms}}\left(\mathrm{H}_{2}-\mathrm{AC}(\mathrm{GC})\right)>\mathrm{n}_{\mathrm{ms}}\left(\mathrm{H}_{2}-\mathrm{AC}(\mathrm{CS})\right)$. This behavior could be mainly explained by the existence of a variety of functional groups on the surfaces of the AC (GC) adsorbents, which could expedite and facilitate the binding of the hydrogen molecules via specific interac- 


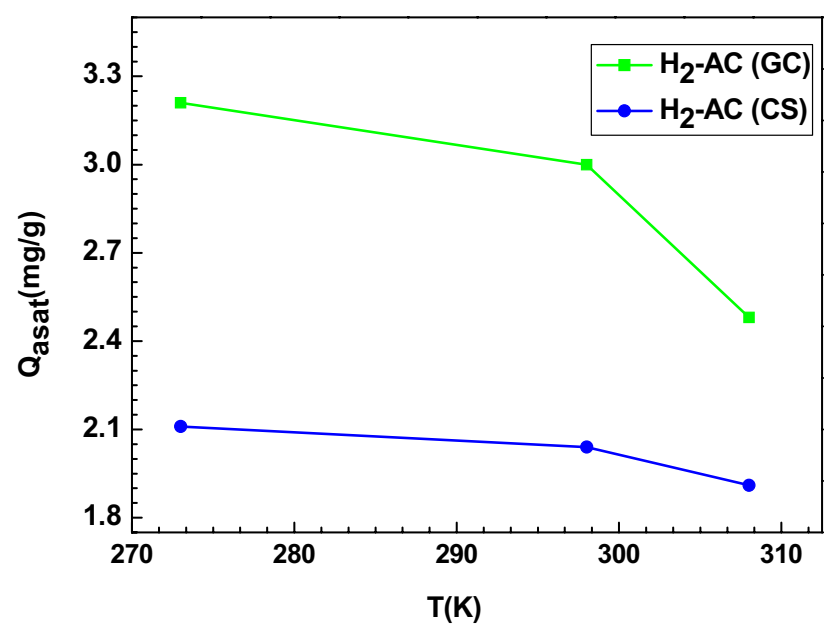

Figure 2. Effect of temperature on the parameter $\mathrm{Q}_{\text {asat }}$ for the adsorption of hydrogen on $\mathrm{AC}(\mathrm{GC})$ and $\mathrm{AC}$ (CS) adsorption system.

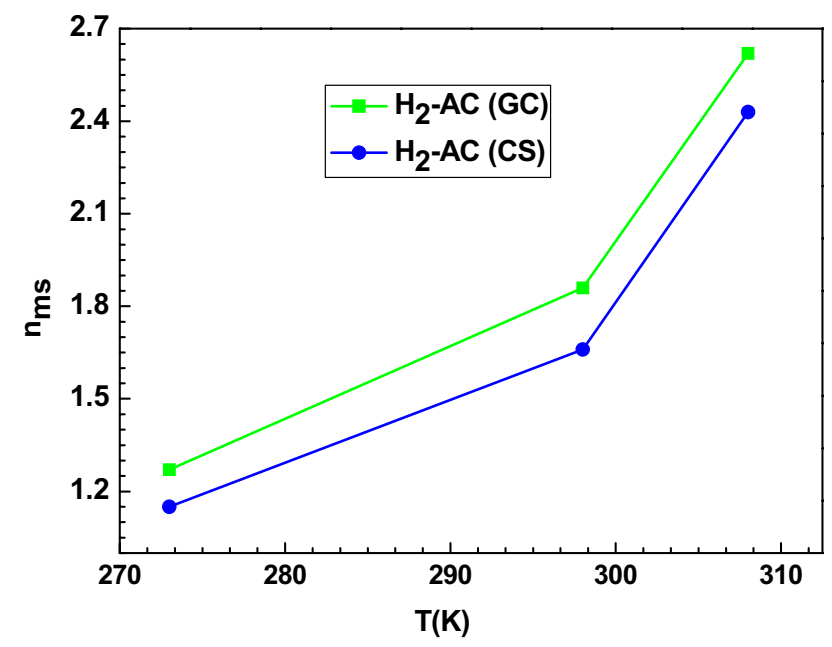

Figure 3. Effect of temperature on the parameter $n_{m s}$ for the adsorption of hydrogen on $A C(G C)$ and $A C$ (CS) adsorption system.

tions with the lead functional groups responsible of the hydrogen adsorption. In this respect, more molecules per site were captured by the AC (GC) adsorbent, affirming by means of statistical physics, that this adsorbent is more adequate for hydrogen storage. From Fig. 3, we notice that the number of captured hydrogen molecules per binding site is slightly increased as a function of temperature for both AC adsorbents. This behavior is due to the thermal agitation.

The receptor site density $\left(D_{s r}\right) . \quad D_{s r}$ is a useful parameter which provides indication on the density distribution of receptor sites available to the hydrogen molecules. It expresses the capacity of the AC adsorbents' surface to capture the hydrogen molecules. The evolution of the density of the receptor sites with respect to temperature is illustrated in Fig. 4. It can be observed that this parameter varies in an opposite manner with the temperature. The opposite trend of $D_{s r}$ expresses an antagonistic effect. Indeed, both $n_{m s}$ and $D_{s r}$ give an indication about the hydrogen adsorption amount on the AC adsorbents and they are not independent of each other. When the number of hydrogen molecules rises, this creates a steric hindrance for the filling of the corresponding $\mathrm{D}_{\text {sr }}$ spots, and therefore $\mathrm{D}_{\mathrm{sr}}$ tends to decrease. This evidence can be explained by thermal agitations, which characterize a normal adsorption mechanism described by the negative binding energy and the exothermicity of the mechanism, due to the adsorption energy affected by the thermal collisions.

The total number of formed layers $\left(\mathrm{N}_{\mathrm{T}}=1+\mathrm{N}\right)$. The physicochemical parameter $\mathrm{N}_{\mathrm{T}}$ is the global number of hydrogen layers adsorbed on both AC adsorbents and its calculated values are given in Table 3 and illustrated in Fig. 5. It should be noted that the created layers varied from 3.42 to 4.13 and from 2.87 to 3.75 for systems $\mathrm{H}_{2}-\mathrm{AC}$ (GC) and $\mathrm{H}_{2}-\mathrm{AC}(\mathrm{CS})$, respectively. This theoretical evidence indicates that the adsorption of hydrogen on the 


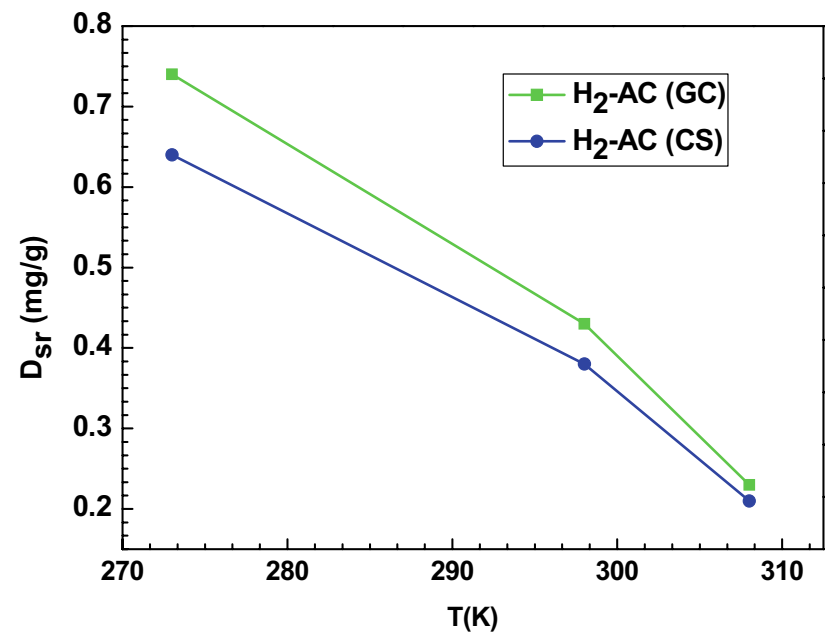

Figure 4. Effect of temperature on the parameter $D_{\text {sr }}$ for the adsorption of hydrogen on $A C$ (GC) and AC (CS) adsorption system.

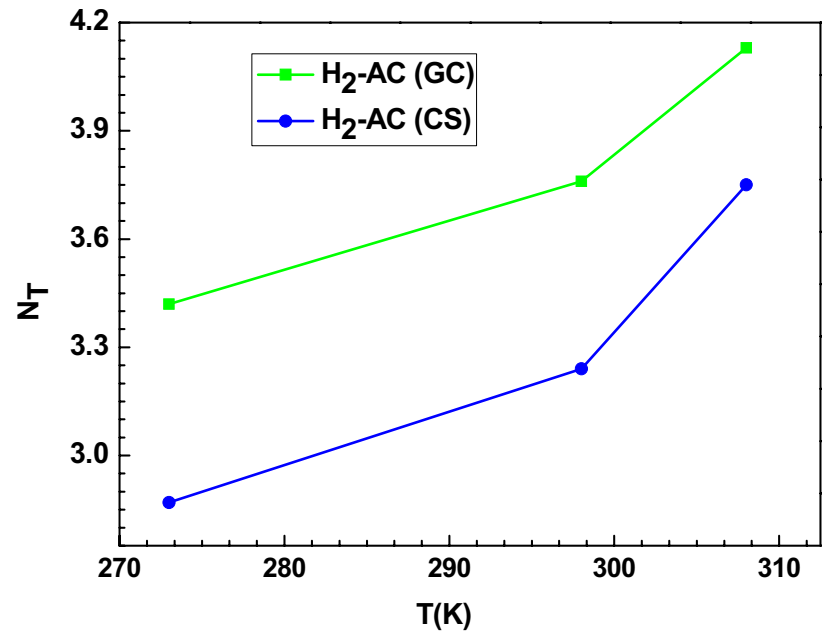

Figure 5. Effect of temperature on the parameter $\mathrm{N}_{\mathrm{T}}$ for the adsorption of hydrogen on $\mathrm{AC}(\mathrm{GC})$ and $\mathrm{AC}(\mathrm{CS})$ adsorption system.

AC (GC) is practically done by the creation of four layers and by the creation of three or almost four hydrogen layers for the AC (CS) system, rising with the operating temperature. For instance, at $\mathrm{T}=308 \mathrm{~K}$, the $\mathrm{N}_{\mathrm{T}}$ value is equal to 3.75. This value indicates that three or four layers are constituted of hydrogen molecules on AC (CS). It can be analyzed by the respective proportions $(\mathrm{z})$ and $(1-\mathrm{z})$ or numerically by $25 \%$ of the hydrogen molecules created three layers and $75 \%$ of the hydrogen molecules constituted four layers. Hence, the four layers are not totally occupied. According to the behavior of $\mathrm{N}_{\mathrm{T}}$ as a function of temperature (Fig. 5), it can be deduced that the normal thermal agitation relatively improved the creation of layers for the hydrogen adsorption on both ACs.

Energetic analysis: study of the adsorption energies. An energetic study is significant to describe the interactions between the hydrogen molecules and the hydrogen molecules with the AC adsorbents. Referring to the expression of the MLS model, two pressures at half saturation $\mathrm{P}_{\mathrm{hs} 1}$ and $\mathrm{P}_{\mathrm{hs} 2}$ are associated to the binding energies of various sorbed layers. From these two parameters $\left(\mathrm{P}_{\mathrm{hs} 1}\right.$ and $\left.\mathrm{P}_{\mathrm{hs} 2}\right)$, we can estimate the values of the two adsorption energies and hence interpret their variation with respect to temperature. The formulas of these energies are written as:

$$
\begin{aligned}
& -\varepsilon_{1}=-k_{B} \mathrm{~T} \ln \frac{\mathrm{P}_{\mathrm{vs}}}{\mathrm{P}_{\mathrm{hs} 1}} \\
& -\varepsilon_{2}=-k_{B} \mathrm{~T} \ln \frac{\mathrm{P}_{\mathrm{vs}}}{\mathrm{P}_{\mathrm{hs} 2}}
\end{aligned}
$$




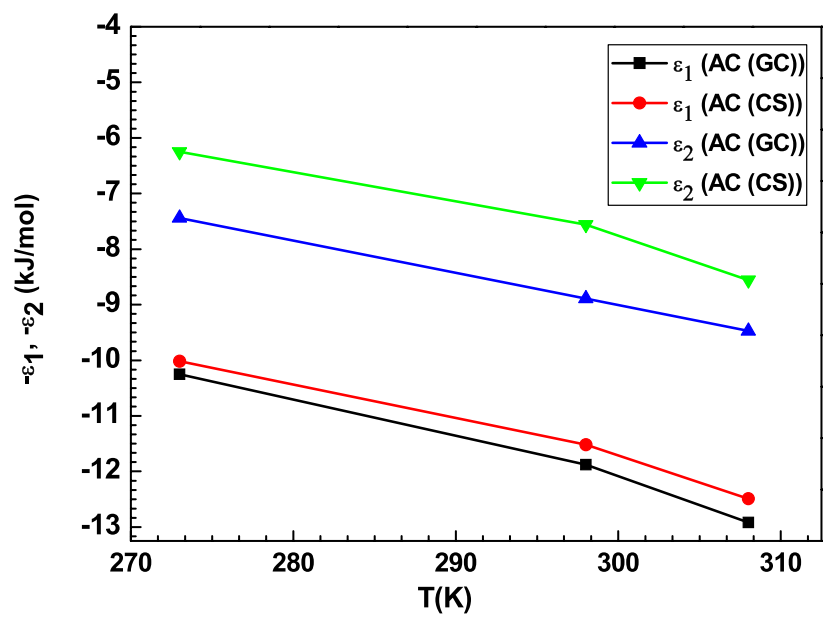

Figure 6. Effect of temperature on the hydrogen adsorption energy $\left(-\varepsilon_{1}\right.$ and $\left.-\varepsilon_{2}\right)$ of AC (GC) and AC (CS) adsorption system.

where $\mathrm{k}_{\mathrm{B}}$ is the Boltzmann constant and $\mathrm{P}_{\mathrm{vs}}$ is the saturated vapor pressure of hydrogen at the corresponding temperature.

Figure 6 illustrates the evolution of the adsorption energies $-\varepsilon_{1}$ and $-\varepsilon_{2}$ with respect to temperature. The adsorption energies values estimated in this paper are about $6-12 \mathrm{~kJ} / \mathrm{mol}$ for the two AC adsorbents (Table 3 ) and are comparable to many other works ${ }^{9,20-23}$. These values indicate that binding between hydrogen and the AC surface takes place via a physical adsorption. According to Fig. 6, it can be observed that the adsorption energy decreases when the temperature increases, which was associated to the reduction of the uptake capacity in agreement with the exothermic nature of the hydrogen adsorption process.

Evaluation of the thermodynamic functions. The thermodynamic functions can be estimated using a general analysis of the hydrogen adsorption system with the theoretical formula of the multilayer statistical physics model. The entropy, the Gibbs free energy and the internal energy were estimated and interpreted in the following subsections.

Entropy. The study of the entropy has a significant interest because it provides details about the order and disorder of the hydrogen molecules on the AC adsorbent surface. The analytical equation of the adsorption entropy can be derived using the grand potential and the selected grand canonical partition function as follows ${ }^{24}$ :

$$
\begin{gathered}
J=-k_{B} T \ln Z_{g c} \\
J=-\frac{\partial \ln Z_{g c}}{\partial \beta}-T_{a}
\end{gathered}
$$

By equalizing these two expressions, we obtain the following equation:

$$
\frac{\mathrm{S}_{\mathrm{a}}}{\mathrm{k}_{\mathrm{B}}}=-\beta \frac{\partial \ln Z_{\mathrm{gc}}}{\partial \beta}+\ln \mathrm{Z}_{\mathrm{gc}}
$$

The entropy variation as a function of the hydrogen pressure is illustrated in Fig. 7. From this figure, it can be globally observed that the entropy presents two different trends below and above the half saturation pressure values $\left(\mathrm{P}_{\mathrm{hs} 1}\right)$ and $\left(\mathrm{P}_{\mathrm{hs} 2}\right)$. In fact, the entropy curves rise with the pressure before the half-saturation and decrease after this point. When the adsorption pressure is lower than $\mathrm{P}_{\mathrm{hs}}$, the hydrogen molecules have a high probability to select a binding site to adsorb and hence the disorder rises at the AC (GC) surface with the pressure. After the half-saturation, the hydrogen molecules have few possibilities to select the adsorbent site since the AC (GC) surface tends to the saturation and hence tends towards the order. The entropy can reach zero when the saturation is attained.

Gibbs free energy. The Gibbs free energy characterizes the spontaneity of the adsorbed systems. The behavior of the external environment was integrated and estimated by the following expression ${ }^{24}$ :

$$
\mathrm{G}=\mu \mathrm{Q}_{\mathrm{a}}
$$

where $\mathrm{Q}_{\mathrm{a}}$ is the sorbed quantity and $\mu$ is the chemical potential of the receptor site.

The estimated values of the Gibbs free energy, illustrated in Fig. 8, are negative, demonstrating that the sorption process occurred spontaneously. In addition, the Gibbs free energy values decreased in module with the 


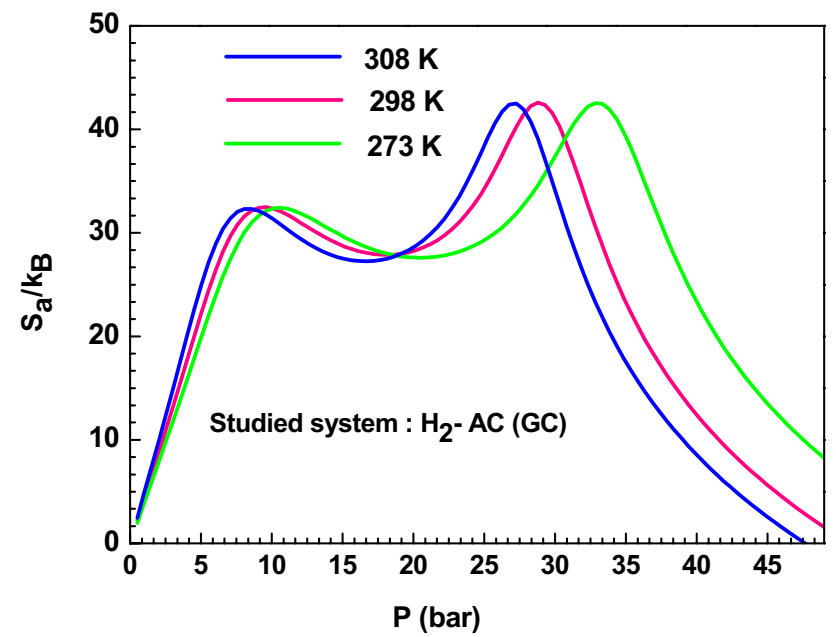

Figure 7. Variation of the entropy versus pressure for the AC (GC) adsorption system at different temperature.

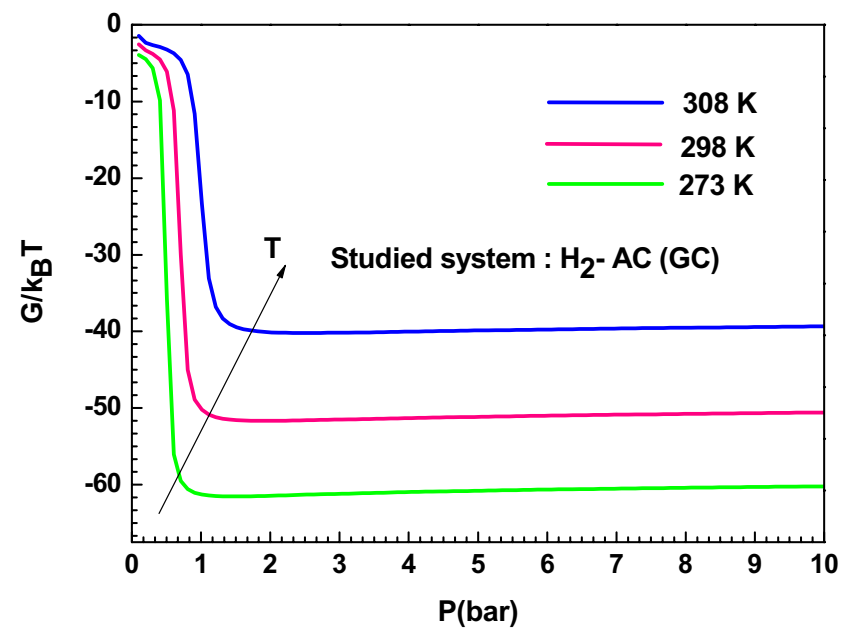

Figure 8. Variation of the free enthalpy versus pressure for the AC (GC) adsorption system at different temperature.

increasing temperature for AC (GC) adsorbent, which demonstrates a reduction in adsorption feasibility at high temperatures. The temperatures prevent the hydrogen adsorption mechanism from occurring.

Internal energy. The internal energy is estimated using the following expressions ${ }^{24}$ :

$$
\begin{gathered}
\mathrm{E}_{\mathrm{int}}=\sum \varepsilon_{\mathrm{i}} \mathrm{N}_{\mathrm{i}} \\
\mathrm{E}_{\mathrm{int}}=-\frac{\partial \ln \mathrm{Z}_{\mathrm{gc}}}{\partial \beta}+\frac{\mu}{\beta}\left(\frac{\partial \ln \mathrm{Z}_{\mathrm{gc}}}{\partial \mu}\right)
\end{gathered}
$$

The variation of this potential function is depicted in Fig. 9. It can be noticed that the $\mathrm{E}_{\mathrm{int}}$ values are negative, affirming that the $\mathrm{AC}(\mathrm{GC})$ hydrogen adsorption system evolve spontaneously because they release energy. Also, it can be observed that $\mathrm{E}_{\text {int }}$ increases algebraically but decreases in module when the temperature rises, and this is mainly due to the thermal agitation.

\section{Conclusion}

The established multilayer model with saturation reproduces much more accurately the experimental equilibrium data, compared to the tested models. The adsorption isotherms of hydrogen on the two ACs based adsorbents were explained and discussed in light of this statistical physics model, at three operating temperatures. The analysis of the number of hydrogen molecules per binding site for the $\mathrm{H}_{2}-\mathrm{AC}(\mathrm{GC})$ and $\mathrm{H}_{2}-\mathrm{AC}(\mathrm{CS})$ systems indicates 


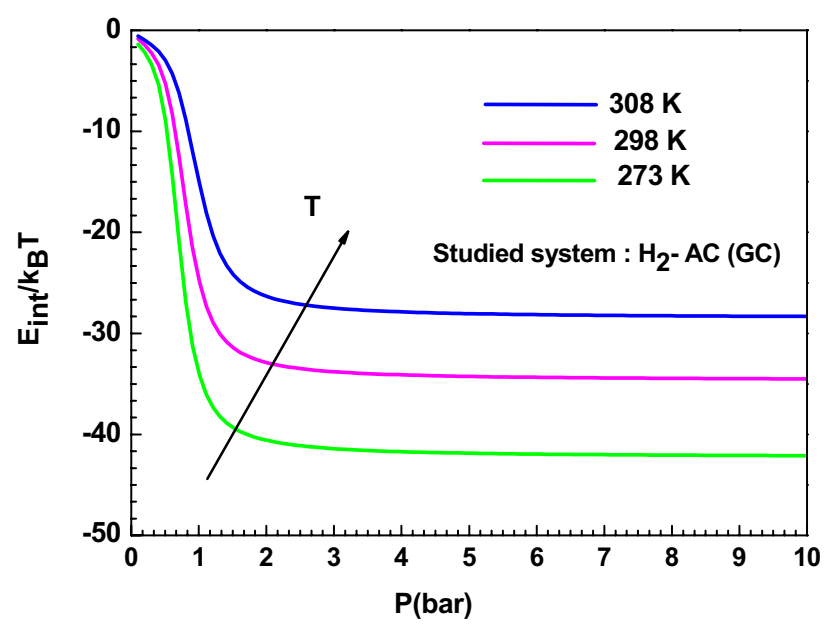

Figure 9. Variation of the internal energy versus pressure for the AC (GC) adsorption system at different temperature.

that the hydrogen molecules could be aggregated in a monomer $\left(\mathrm{n}_{\mathrm{ms}}=1\right)$ and a dimmer $\left(\mathrm{n}_{\mathrm{ms}}=2\right)$ form on the receptor sites. The hydrogen aggregation degree rose with the increasing temperature, demonstrating that the two ACs adsorbents were energetically activated. The increase of temperature has conducted to the reduction in the density of the receptor sites for both tested adsorbents due to thermal agitation. The study of the adsorbed amount at saturation indicates that the quite temperature is the perfect condition to get a good storage of hydrogen on the AC adsorbents. The estimated values of the adsorption energies indicate that hydrogen is physisorbed. To describe the sorption mechanism, the entropy was estimated according to the selected multilayer model. The variation of the internal energy and the Gibbs free energy indicates that the hydrogen adsorption on both AC adsorbents is spontaneous till the saturation is reached.

Received: 2 June 2020; Accepted: 24 August 2020

Published online: 30 September 2020

\section{References}

1. Foong, S. Y. et al. Valorization of biomass waste to engineered activated biochar by microwave pyrolysis: progress, challenges, and future directions. Chem. Eng. J. 389, 124401 (2020).

2. Sethia, G. \& Sayari, A. Activated carbon with optimum pore size distribution for hydrogen storage. Carbon 99, 289-294 (2016).

3. Mananghaya, M. R., Santos, G. N. \& Yu, D. Hydrogen adsorption of Ti-decorated boron nitride nanotube: a density functional based tight binding molecular dynamics study. Adsorption. 7, 1-8 (2018).

4. Mananghaya, M., Belo, L. P. \& Beltran, A. Adsorption of hydrogen in Scandium/Titanium decorated nitrogen doped carbon nanotube. Mater. Chem. Phys. 180, 357-363 (2016).

5. Mananghaya, M. R. A simulation of hydrogen adsorption/desorption in metal-functionalized BN nanotube. Mater. Chem. Phys. 240, 122159 (2020).

6. Wróbel-Iwaniec, I., Díez, N. \& Gryglewicz, G. Chitosan-based highly activated carbons for hydrogen storage. J. Hydrog. Energy. 40, 5788-5796 (2015).

7. Gryglewicz, G. \& Lorenc-Grabowska, E. Mesoporous activated carbons from Ca and Fe exchanged sub-bituminous and bituminous coals. Carbon 42, 688-691 (2004).

8. Schobert, H. H. \& Song, C. Chemicals and materials from coal in the 21st century. Fuel 81, 15-32 (2002).

9. Feng, Y., Wang, J., Liu, Y. \& Zheng, Q. Adsorption equilibrium of hydrogen adsorption on activated carbon, multiwalled carbon nanotubes and graphene sheets. Cryogenics 101, 36-42 (2019).

10. Üner, O. Hydrogen storage capacity and methylene blue adsorption performance of activated carbon produced from Arundo donax. Mater. Chem. Phys. 237, 121858 (2019).

11. Nasruddin, M., Martin, A., Alhamid, M. I. \& Tampubolon, D. Adsorption isotherms of hydrogen on granular activated carbon coal derived and coconut shell derived. Heat Transf. Eng. 38, 403-408 (2017).

12. Ben Lamine, A. \& Bouazra, Y. Application of statistical thermodynamics to the olfaction mechanism. Chem. Senses. 22, 67-75 (1997).

13. Knani, S., Mathlouthi, M. \& Ben Lamine, A. Modeling of the psychophysical response curves using the grand canonical ensemble in statistical physics. J. Food Biophys. 2, 183-192 (2007).

14. Khalfaoui, M., Knani, S., Hachicha, M. A. \& Ben Lamine, A. New theoretical expressions for the five adsorption type isotherms classified by BET based on statistical physics treatment. J. Colloid Interfaces Sci. 263, 350-356 (2003).

15. Diu, B., Guthmann, C., Lederer, D. \& Roulet, B. Macroscopic motion of a totally isolated system in statistical equilibrium. Am. J. Phys. 58, 974-978 (1990).

16. Khalfaoui, M., Baouab, M. H. V., Gauthier, R. \& Ben Lamine, A. Statistical physics modelling of dye adsorption on modified cotton. Adsorpt. Sci. Technol. 20, 17-31 (2002).

17. Ben Yahia, M. et al. On the statistical physics of finite multilayer adsorption model on gaseous phase, in The fifth International Renewable Energy Congress IREC. 1-5 (2014)

18. Yazidia, A. et al. Monolayer and multilayer adsorption of pharmaceuticals on activated carbon: application of advanced statistical physics models. J. Mol. Liq. 283, 276-286 (2019).

19. Bouaziz, N., Manaa, M. B., Aouaini, F. \& Ben Lamine, A. Investigation of hydrogen adsorption on zeolites A, X and Y using statistical physics formalism. Mater. Chem. Phys. 225, 111-121 (2019). 
20. Zhou, L., Zhou, Y. \& Suna, Y. A comparative study of hydrogen adsorption on superactivated carbon versus carbon nanotubes. J. Hydrog. Energy. 29, 475-479 (2004).

21. Zhao, X. B., Xiao, B., Fletcher, A. J. \& Thomas, K. M. Hydrogen adsorption on functionalized nanoporous activated carbons. J. Phys. Chem. B 109, 8880-8888 (2005).

22. Cheng, H. S. et al. Molecular dynamics simulations on the effects of diameter and chirality on hydrogen adsorption in single-walled carbon nanotubes. J. Phys. Chem. B. 10, 93780 (2005).

23. Purewal, J. J., Kabbour, H., Vajo, J. J., Ahn, C. C. \& Fultz, B. Pore size distribution and supercritical hydrogen adsorption in activated carbon fibers. Nanotechnology 20, 204012 (2009).

24. Couture, L. \& Zitoun, R. Physique Statistique (Ellipses, Paris, 1992).

\section{Acknowledgements}

This project was funded by the Deanship of Scientific Research (DSR), King Abdulaziz University, Jeddah, under Grant No. (D-135-662-1441). The authors, therefore, gratefully acknowledge DSR technical and financial support.

\section{Author contributions}

Credit Author Statement: M.B.Y.: Conceptualization, writing_original draft, S.W.: Data curation and Resources, Software.

\section{Competing interests}

The authors declare no competing interests.

\section{Additional information}

Correspondence and requests for materials should be addressed to M.B.Y. or S.W.

Reprints and permissions information is available at www.nature.com/reprints.

Publisher's note Springer Nature remains neutral with regard to jurisdictional claims in published maps and institutional affiliations.

(c) (i) Open Access This article is licensed under a Creative Commons Attribution 4.0 International License, which permits use, sharing, adaptation, distribution and reproduction in any medium or format, as long as you give appropriate credit to the original author(s) and the source, provide a link to the Creative Commons licence, and indicate if changes were made. The images or other third party material in this article are included in the article's Creative Commons licence, unless indicated otherwise in a credit line to the material. If material is not included in the article's Creative Commons licence and your intended use is not permitted by statutory regulation or exceeds the permitted use, you will need to obtain permission directly from the copyright holder. To view a copy of this licence, visit http://creativecommons.org/licenses/by/4.0/.

(c) The Author(s) 2020 